\title{
GENERALISED QUASIPERFECT NUMBERS
}

\section{GRaeme L. Cohen}

The main concern of this thesis is an investigation of the equation $\sigma(n)=2 n+k^{2}$, where $n$ and $k$ are relatively prime positive integers, $k$ is odd and $\sigma(n)$ is the sum of the positive divisors of $n$. We have called any $n$ which satisfies this equation for some $k$ a generalised quasiperfect number, or, more specifically, a $k$-quasiperfect number, since such $n$, for $k=1$, are known as quasiperfect numbers. No quasiperfect number has yet been found, but neither has a proof that none exist. The same is true of generalised quasiperfect numbers: we know of no example, for any $k$, nor can we prove for any $k$ that none exist.

Nonetheless, we have been able to establish a great number of necessary conditions for the existence of a generalised quasiperfect number, and many of these are direct generalisations of previously known results about quasiperfect numbers. For example, the first necessary property of a quasiperfect number to be established was that such a number must be an odd perfect square; the same is true of a $k$-quasiperfect number, for every $k$.

The following definitions are pertinent to the ensuing summary. For a real number $\alpha \geq 1$, we say that a positive integer $n$ is $\alpha$-perfect if $\sigma(n)=\alpha n$, and primitive $\alpha$-abundant if $\sigma(n)>\alpha$ with $\sigma(m)<\alpha m$ for any proper divisor $m$ of $n$. When $\alpha=2$, the prefix is omitted.

Some of the main results of this thesis are as follows. Suppose there is a $k$-quasiperfect number $n$. Then $n$ must have at least four distinct. prime factors, and must exceed $10^{20}$. If $n$ has only four or five

Received I November 1982. Thesis submitted to University of New South Wales, June 1982. Degree approved: October 1982. Supervisor: Dr J.H. Loxton. 
distinct prime factors, then $k$ must exceed $10^{10}$. If $k \leq 44366047$, then $n$ must be primitive abundant. If $n$ is primitive abundant, then $n$ must have at least six distinct prime factors. Much of what was known for the special case of quasiperfect numbers, prior to 1979 , is included in these results.

The thesis includes also a more general investigation of primitive $\alpha$-abundant numbers. We show that, for positive integers $x, y, z$, if $x(n)=y n+z, n$ is not primitive $(y / x)$-abundant and $n / s$ is not $(y / x)$-perfect (where $s^{d}$ is the largest prime power in $n$ ), then

$$
n<\max \left\{2(z-1)^{5 / 2} / y, 4\left(z+\frac{1}{2}\right)^{3} / 27 y\right\} .
$$

We table all solutions of the equation $\sigma(n)=y n+z \quad(y \geq 2, z \leq 210)$ for which $n$ is not primitive $y$-abundant. These extend previous results in the literature. Some new properties of a primitive $\alpha$-abundant number $n$ are obtained: $\sigma(n) / n<\alpha+\min \left\{\frac{1}{2}, 3 \alpha e^{-5 \alpha / 9} / 2\right\}$ and $\sigma(n) / n<\alpha+1.6 \alpha / \log n$. We list all primitive abundant numbers $n$ with $\sigma(n) / n \geq 2.05$ and, in so doing, show that $\sigma(n) / n<2.16104$.

The first result of the preceding paragraph has a useful application to generalised quasiperfect numbers. We use it in showing that, if $n$ is $k$-quasiperfect and not primitive abundant, then $n<2\left(1+10^{-15}\right) k^{6} / 27$. This makes use of a result developed in connection with the existence of odd perfect numbers and, to our knowledge, is the first application of that theory outside the field itself.

It was conjectured in 1975 that the equation $\sigma(n)=2 n+k^{2}$, with $k$ odd, has no solutions at all. We show this is not true, and exhibit seven solutions. (The smallest $n$ for which $\sigma(n)=2 n+k^{2}$ for some odd $k$ is $n=2^{2} 7^{2} 53^{2}$.) The final chapter of the thesis considers this and other aspects of the equation $\sigma(n)=2 n+k^{2}$ when some of our original. restrictions on $n$ and $k$ are relaxed.

In the Appendix, some new results are proved concerning odd perfect numbers. Suppose $N=p^{a} q_{1}^{a b}$ i. $\ldots q_{u}^{2 b} u$ (where $p \equiv a \equiv 1(\bmod 4)$ and 
$p, q_{1}, \ldots, q_{u}$ are distinct odd primes) is an odd perfect number. We prove the following, which are extensions of earlier results in the literature. If $b_{1}=\ldots=b_{u}=b$, then $b \neq 6,8,11,14$ or 18 (a joint result with R.J. Williams). If $b_{2}=\ldots=b_{u}=1$, then $b_{1} \neq 5$ or 6. If $x$ is the number of powers $q_{i}^{2 b}$ in which both $q_{i} \equiv 1(\bmod 4)$ and $b_{i} \equiv I(\bmod 2)$, then $p-a \equiv 4 x(\bmod 8)$. If $b_{1}=\ldots=b_{t}=1$ and $b_{t+1}=\ldots=b_{u}=2$, then $1916 \leq u-t \leq \sqrt{a}+2 t$ and $N>10^{83282}$. If $b_{2}=2$ and $b_{3}=\ldots=b_{u}=1$, then $b_{1} \neq 3$.

School of Mathematical Sciences, New South Wales Institute of Technology, Post Office Box 123, Broadway, New South Wales 2007, Australia. 